\title{
Clinical and Pathologic Evaluation of Adnexal Torsion Patients in Adolescence, Reproductive and Postmenopausal Periods
}

\author{
Bora Coşkun $^{1}$, Burcu Timur ${ }^{2}$, Buğra Coşkun ${ }^{1}$, Ferdi Kınc1 ${ }^{3}$, Coşkun Şimşirir ${ }^{1}$ \\ ${ }^{1}$ Liv Hospital Ankara Department of Obstetrics and Gynecology, Ankara, Turkey \\ ${ }^{2}$ Ordu University Faculty of Medical Department of Obstetrics and Gynecology, Ordu, Turkey \\ ${ }^{3}$ Muğla Sttk1 Koçman Univercity Training and Research Hospital Department of Obstetrics and Gynecology
}

Received: 30 April 2019, Accepted: 24 May 2019, Published online: 28 August 2019

(C) Ordu University Institute of Health Sciences, Turkey, 2019

\begin{abstract}
Objective: The objective of our study was to compare the adnexal torsion patients in different age groups clinically and pathologically.

Methods: Sixty-eight patients who had applied with pelvic pain and adnexal torsion diagnosis had been confirmed intraoperatively were included in the study. Patients were divided into three groups based on their ages as the adolescent period (Group 1), reproductive period (Group 2) and postmenopausal period (Group 3). Ages, history of torsion, history of past operations, periods between the date of hospitalization and operation, periods of hospital stay, adnexa as the localization of torsion and number of tours of torsion were examined in groups. Mass lesions found during the operation and pathologic consequences of such masses were examined retrospectively and compared.

Results: No statistically significant differences in torsion history, past operations, WBC and platelet values, neutrophil/lymphocyte ratios, preoperative fever, preoperative nausea and vomiting and preoperative Doppler US findings between the groups included in the studies $(p>0,05)$. Although not statistically significant, WBC values were higher in the postmenopausal patient group, and it was notable that symptoms including fever, nausea and vomiting were absent in this group. The adolescent age group was the group with the least confirmation of torsion diagnosis following the operation with 13 (52\%) patients. Upon evaluation of the operations carried out on patients, it was seen that mostly fertility preserving approaches were preferred for adolescent and reproductive patients with $88 \%$, while more radical methods such as USO $(72.7 \%)$ and TAH+BSO (18.2\%) were preferred in postmenopausal patients. Ovarian tissue with torsion without any pathologic findings is more frequent in the adolescent period as compared to other groups (48\%). In the postmenopausal period however, some pathology was found in the entire portion of the adnexa with torsion. In the postoperative evaluation of the pathology results of patients, no significant differences were observed as regards the diameters of cysts (p:0,207). There are significant differences between the histopathological types.

Conclusion: Although statistically significant differences have not been observed as regards the clinic al presentation and laboratory findings based on age groups; it must be kept in mind that a mass lesion can accompany the torsion and the pathology of this mass can vary. Torsion must be remembered in pelvic pain in the adolescent age group because of the indistinct clinical findings and inadequacy of imaging methods. Key words: Adnexal Torsion, Pelvic Pain, Gyneacologic Emergency
\end{abstract}

Suggested Citation: Coskun B, Timur B, Coskun B, Kinci F, Simsir C. Clinical and Pathologic Evaluation of Adnexal Torsion Patients in Adolescence, Reproductive and Postmenopausal Periods. Middle Black Sea Journal of Health Science 2019; 5(2): 85-92 
Address for correspondence/reprints:

Bora Çoşkun

Telephone number: +90 (532) 7672480

E-mail: drboracoskun@gmail.com

DOI: $10.19127 / \mathrm{mbsjohs.559446}$

\section{Introduction}

Adnexal torsion constituting about 2.5-7.4\% of patients applying to emergency room is defined as the partial or complete rotation of the ovaries, Fallopian tubes or both on the ligamentous supports and vascular pedicles (Hibbard, 1985). The reason for adnexal torsion being one of the most important emergencies of gynecology is the ischemia and necrosis developing as a consequence of the reduced blood flow (Hibbard, 1985). While adnexal torsion can be seen in every age group from intrauterine life to postmenopausal period, it faces us most frequently in the reproductive period (Günay et al., 2018). The diagnosis and treatment is an issue that maintains its actuality based on the facts that it is seen in adolescents and women in reproductive ages (mean age 26 years) and its adverse effects on ovarian functions (Hibbard, 1985).

Conditions leading to adnexal torsion are not clearly known; however, the most common predisposing factor is adnexal mass lesions. Functional ovarian cysts and benign cystic teratoma are frequently seen among the causes of adnexal torsion while malignant cysts and endometrioma are seen less frequently (Huchon and Fauconnier, 2010). Furthermore, the first trimester of pregnancy and assisting reproductive techniques are the other predisposing factors of adnexal torsion (Houry and Abbott, 2001). Hydrosalpinx, hematosalpinx, paraovarian cysts are among the other risk factors of isolated tubal torsion (Ekmekci et al., 2010; Noviello et al., 2018).

Ischemia related to torsion and loss of ovarian functions in relation with the former is the most important complication of torsion, and such adverse effects are reduced significantly in patients who are diagnosed in the early period (Huchon and Fauconnier, 2010). This is particularly important for young patients with fertility expectations
(Santra et al., 2018). At the same time, diagnosis is difficult because of the clinical presentation mostly involving nonspecific findings.

Abdominal pain, nausea, vomiting and fever are typical for the clinical presentation of adnexal torsion. Pain may not be present in every patient, and this causes delays in diagnosis (Bodur et al., 2016). Possibility of necrosis increases in patients who are taken to operation about 10 hours later than the onset of pain (Mazouni et al., 2005). Nausea and vomiting are most common, and can be seen in $70 \%$ of patients (Lomano et al., 1970). Fever, which is an indicator of necrosis, can also be seen as a symptom (Houry and Abbott, 2001). There is no specific laboratory findings; however, leukocytosis is seen frequently (Chiou et al., 2007). The most commonly used imaging technique is ultrasonography (USG). However, according to studies, diagnostic benefit of US is quite limited. This benefit ranges between $46 \%$ and $77 \%$ according to one study, and it has not been possible to show the diagnostic benefit of Doppler US in addition to conventional US (Albayram and Hamper, 2001; Mashiach et al., 2011; Wilkinson and Sanderson, 2012). MRI and CT studies are useful in cases where an accompanying mass lesion is present.

The value of this study lies in the fact that studies comparing the adnexal torsion cases seen in adolescents, adults and postmenopausal individuals are lacking in the literature. In this context, our study will be the first. We aimed at comparing the adnexal torsion cases in different age groups in clinical and pathologic aspects.

\section{Methods}

Files of patients operated due to adnexal torsion in Liv Hospital Ankara between January 2016 and August 2018 were evaluated retrospectively. Ethical approval for the study was obtained from the Clinical Research Ethics Committee of Liv Hospital Ankara. Demographic data, past torsion histories, clinical presentations, findings in operations, post-operative periods and pathology results of patients were reviewed on the computer system and patient files.

Sixty-eight patients applied with pelvic pain and diagnosis of adnexal torsion confirmed intraoperatively were included in the study. All operations conducted on patients included in the study were performed by gynecologists and obstetricians. Patients were divided into three groups according to their ages, namely, the adolescent period (Group 1), reproductive period 
(Group 2) and the postmenopausal period (Group 3). Ages, history of torsion, history of past operations, periods between the date of hospitalization and operation, periods of hospital stay, adnexa as the localization of torsion and number of tours of torsion were examined in groups. Mass lesions found during the operation and pathologic consequences of such masses were examined and compared retrospectively.

Groups were compared in terms of the types and times of operations performed on patients, preoperative symptoms, laboratory markers including leukocyte (WBC) and platelet counts and neutrophil/leukocyte ratio and absence of Doppler flow suggesting torsion.

Mean value, standard deviation, the lowest and the highest median values, frequencies and ratios were used in the descriptive statistics of the data. Distribution of values were measured with Kolmogorov-Simirnov test. ANOVA (Tukey test) and Kruskal-Wallis test were used in the analysis of quantitative data. Chi-square test was used for the analysis of qualitative data; and if the conditions required for the chi-square test could not be met, Fischer test was used for the same. SPSS 22.0 program was used for the analyses. $\mathrm{P}$ value $<0.05$ was accepted as the limit of significance in all analyses.

\section{Results}

Demographic characteristics of patients included in the study are summarized in Table 1. No statistically significant differences were found between the past torsion histories, past operations, WBC and platelet counts, Neutrophil/Lymphocyte ratios, preoperative fever, preoperative nausea and vomiting, and absence of Doppler flow in the adolescent, reproductive and postmenopausal period groups. ( $\mathrm{p}$ value $<0,05$ was accepted as the level of statistical significance.) (Table 1)

Upon evaluation of patients based on their age groups, higher WBC values in the postmenopausal group is notable, although not statistically significant. In the evaluation of the preoperative fever, this was more marked only in the adolescent group, again not statistically significant $(12 \%)$. Furthermore, absence of symptoms including fever, nausea and vomiting in the postmenopausal patient groups is notable. US findings suggesting torsion were seen in the preoperative Doppler US study in the adolescent age group only with a ratio of $32 \%$. Preoperative diagnoses are given in Table 1 based on age groups. The adolescent age group is the one that torsion diagnosis was the least confirmed intraoperatively with 13 patients (52\%). In addition, total of 51 patients were operated with the diagnosis of preliminary torsion in all the age groups within the same period, and torsion diagnosis had become definite in 44 patients $(86 \%)$.

No significant differences were observed in the operation time, period between admission and operation, side of the torsion, number of tours of torsion and hospital stay periods in the intraoperative evaluation of patients. (Table 2) Upon evaluation of the procedures performed on patients however, it was found that fertilitypreserving approaches had been more commonly preferred in the adolescent and reproductive patients $(88 \%)$, while more radical methods had been preferred such as USO (72.7\%) OR TAH+BSO were preferred in postmenopausal patients. It was observed that the period between admission and performance of the operation was longer in the postmenopausal patients, although not statistically significant $(17,6+20,9 ; 14,3+14,6$; $25,3+22,0$ respectively).

Evaluation of postoperative pathology results of patients showed no significant differences in the diameters of cysts $(p=0,207)$; however, there are significant differences in histopathological types ( $\mathrm{p}$ $=0.015)$. (Table 3$)$ In the histopathological evaluation, mature cystic teratoma (20\%) and simple serous cysts (12\%) were found in the adolescent age group, mature cystic teratoma (21.9\%), mucinous cystadenoma (18.8\%) and simple serous cyst $(18.8 \%)$ in the reproductive age group, and widespread hemorrhagic infarct (63\%) in the postmenopausal age group come to the front. One $(9.1 \%)$ malignant ovarian tumor was found in the postmenopausal age group. Ovarian tissue with torsion and without any other pathologic finding is more frequent in the adolescent period as compared to other groups (48\%). In the postmenopausal period however, some pathology was found in the entire portion of the adnexa with torsion. 
Table 1. Demographic Characteristics and Preoperative Findings of Patients

\begin{tabular}{|c|c|c|c|c|c|}
\hline & & $\begin{array}{l}\text { Adolescence } \\
\text { Period (n:25) }\end{array}$ & $\begin{array}{l}\text { Reproductive } \\
\text { Period (n:32) }\end{array}$ & $\begin{array}{l}\text { Postmenopausal } \\
\text { Periods (n:11) }\end{array}$ & $\mathrm{P}$ \\
\hline Age & & $15,0+1,7$ & $31,4+4,8$ & $52,2+5,9$ & \\
\hline Past torsion history & & $2(\% 8)$ & $4(\% 12,5)$ & $0(\% 0)$ & 0.566 \\
\hline Past operations & $\begin{array}{l}\text { No } \\
\text { USO } \\
\text { Appendectomy } \\
\text { Cesarean } \\
\text { Cystectomy }\end{array}$ & $\begin{aligned} 22 & (\% 88) \\
0 & (\% 0) \\
1 & (\% 4) \\
2 & (\% 8) \\
0 & (\% 0)\end{aligned}$ & $\begin{array}{c}22(\% 68,8) \\
2(\% 6,3) \\
2(\% 6,3) \\
4(\% 12,5) \\
2(\% 6,3)\end{array}$ & $\begin{array}{c}6(\% 54,5) \\
1(\% 9,1) \\
1(\% 9,1) \\
3(\% 27,3) \\
0(\% 0)\end{array}$ & 0,078 \\
\hline $\mathrm{WBC}\left(* 10^{3}\right)$ & & $15,6+27,6$ & $13,7+14,5$ & $22,6+41,7$ & 0,985 \\
\hline Platelet Count $\left(* 10^{3} / \mathrm{mm}\right.$ & & $272,0+53,9$ & $267,2+61,3$ & $279,1+79,5$ & 0,836 \\
\hline Neutrophil/Lymphocyte & atios & $6,8+5,2$ & $5,9+3,7$ & $7,5+4,8$ & 0,519 \\
\hline Preoperative Diagnosis & $\begin{array}{l}\text { Torsion } \\
\text { Adnexal Mass } \\
\text { Rupture } \\
\text { Appendicitis }\end{array}$ & $\begin{array}{c}13(\% 52) \\
6(\% 12) \\
2(\% 8) \\
4(\% 16)\end{array}$ & $\begin{array}{c}25(\% 78,1) \\
4(\% 12,5) \\
3(\% 9,4) \\
0(\% 0)\end{array}$ & $\begin{array}{c}6(\% 54,5) \\
4(\% 36,4) \\
1(\% 9,1) \\
0(\% 0)\end{array}$ & 0.082 \\
\hline Preoperative Fever & & $3(\% 12)$ & $0(\% 0)$ & $0(\% 0)$ & 0,612 \\
\hline Preoperative Nausea-Vor & niting & $3(\% 12)$ & $7(\% 21,9)$ & $0(\% 0)$ & 0,292 \\
\hline Absence of Doppler Flov & & $8(\% 32)$ & $13(\% 40,6)$ & $6(\% 54,5)$ & 0,093 \\
\hline
\end{tabular}


Table 2. Intraoperative Evaluation of Patients

$\begin{array}{llll}\text { Adolescence } & \begin{array}{l}\text { Reproductive Period } \\ \text { Period (n:25) }\end{array} & \begin{array}{l}\text { Postmenopausal } \\ \text { Periods (n:11) }\end{array} & p\end{array}$

\begin{tabular}{|c|c|c|c|c|}
\hline \multicolumn{4}{|l|}{ Operation } & \multirow[t]{6}{*}{0,136} \\
\hline Detorsion & $11(\% 44)$ & $8(\% 25)$ & $0(\% 0)$ & \\
\hline Detorsion + Cyst excision & $11(\% 44)$ & $13(\% 40,6)$ & $0(\% 0)$ & \\
\hline Salpingectomy & $1(\% 4)$ & $1(\% 3,1)$ & $1(\% 9,1)$ & \\
\hline Unilateral Salpingo-oophorectomy & $2(\% 8)$ & $10(\% 31,3)$ & $8(\% 72,7)$ & \\
\hline $\begin{array}{l}\text { Total Abdominal Hysterectomy + } \\
\text { Bilateral Salpingo-oophorectomy }\end{array}$ & $0(\% 0)$ & $0(\% 0)$ & $2(\% 18,2)$ & \\
\hline Duration of Operation (min) & $54,6+29,6$ & $56,3+29,2$ & $70,0+31,1$ & 0,263 \\
\hline $\begin{array}{l}\text { Duration of Admission to Operation } \\
\text { (hours) }\end{array}$ & $17,6+20,9$ & $14,3+14,6$ & $25,3+22,0$ & 0,499 \\
\hline Torsion Side & & & & 0,506 \\
\hline Right & $13(\% 52)$ & $19(\% 59,4)$ & $8(\% 72,7)$ & \\
\hline Left & $12(\% 48)$ & $13(\% 40,6)$ & $3(\% 27,3)$ & \\
\hline Torsion Type & & & & 0,318 \\
\hline Over & $12(\% 48)$ & $12(\% 37,5)$ & $5(\% 45,5)$ & \\
\hline Tubal & $0(\% 0)$ & $1(\% 3,1)$ & $0(\% 0)$ & \\
\hline Adnexa & $13(\% 52)$ & $19(\% 59,4)$ & $6(54,5)$ & \\
\hline Number Of Torsion Cycles & $2,5+1,5$ & $2,3+1,3$ & $2,3+1,3$ & 0,918 \\
\hline Hospitalization Period (hours) & $40,8+26,6$ & $38,0+19,8$ & $38,1+17,7$ & 0,993 \\
\hline
\end{tabular}

Table 3. Postoperative Pathology Results of Patient

\begin{tabular}{lllll}
\hline & $\begin{array}{l}\text { Adolescence Period } \\
(\mathrm{n}: 25)\end{array}$ & $\begin{array}{l}\text { Reproductive } \\
\text { Period (n:32) }\end{array}$ & $\begin{array}{l}\text { Postmenopausal } \\
\text { Periods (n:11) }\end{array}$ & $\mathrm{p}$ \\
\hline Cyst Diameter (Avg. \pm SD; Med.) & $75.6 \pm 24.2 ; 67$ & $89.9 \pm 31.8 ; 86$ & $87.4 \pm 32.9 ; 75$ & 0.207 \\
\hline Cyst Pathology & & & & 0.015 \\
Paratubal cyst + hydrosalpinx & $1(\% 4)$ & $0(\% 0)$ & $1(\% 9.1)$ & $1(\% 9.1)$ \\
Mature Cystic Teratoma & $5(\% 20)$ & $7(\% 21.9)$ & $1(\% 9.1)$ \\
Mucinous Cystadenoma & $2(\% 8)$ & $6(\% 18.8)$ & $7(\% 63.6)$ \\
Common Hemorrhagic İnfarct & $0(\% 0)$ & $4(12.5)$ & $0(\% 0)$ \\
Simple Serous Cyst & $5(\% 20)$ & $6(\% 18.8)$ & $1(\% 9.1)$ \\
Cancer & $0(\% 0)$ & $0(\% 0)$ & $0(\% 0)$
\end{tabular}

\section{Discussion}

Diagnosis and treatment in the early period are important in torsion patients for preservation of organ functions. While clinical symptoms and findings are nonspecific for diagnosis, torsion presents with some signs including nausea, vomiting and pelvic pain and some findings including muscular defense, rebound and tenderness. Clinically, patients commonly apply with abdominal pain with sudden onset. Pain is localized in the lower right quadrant as sharp and stinging pain with sudden onset generally following a physical activity. Fever is added to the clinical picture in some cases. (White and Stella, 2005) In our study, while abdominal or pelvic pain was present in all the patients, nausea and vomiting found 10 patients $(14.7 \%)$ and fever was found in 3 $(4.4 \%)$. Nausea and vomiting were found in none of the postmenopausal patients. Since the clinical findings other than pain rarely accompanies and creates confusion in many other clinical pictures, torsion must be remembered in women with acute lower abdominal pain.

US, Doppler US, magnetic resonance imaging and computerized tomography can be used for the diagnosis of adnexal torsion as assisting imaging 
methods (Chang et al., 2011). B-mode US and Doppler US are imaging methods that should be selected in the first place in patients with suspected torsion based on their advantages of being inexpensive, easily accessible and ability to evaluate the blood flow (Bronstein et al., 2015). The most frequently-seen findings in imaging include the expansion in ovaries and absence of Doppler flow. However, normal blood flow can be seen in partial torsion with preserved arterial blood flow and interrupted lymphatic drainage (Shadinger et al., 2008), which can lead to delayed diagnosis or evaluation based on an erroneous preliminary diagnosis. PPV is reported as $19-35 \%$ for adnexal torsion with transabdominal ultrasound and $94 \%$ with transvaginal ultrasound (Naiditch and Barsness, 2013) Use of TV US was limited for the adolescent age group in our study, 4 patients $(16 \%)$ were operated with the preliminary diagnosis of appendicitis, possibly because of the similarity between the findings and the limitation of the use of imaging methods in this age group. Furthermore, it was found that the positive Doppler finding involving loss of flow consistently with torsion was the lowest in the adolescent period with $32 \%$. (Table 1). CT and MRI can be used as assisting diagnostic tools in the adolescent patient group; however, routine use of these methods in the diagnosis of adnexal torsion is not recommended because of their lower specificities and sensitivities and higher costs.(Moribata et al., 2015) Moreover, in spite of these methods, the rate of confirmation of the diagnosis in operations carried out with the preliminary diagnosis of adnexal torsion is reported between $23 \%$ and $62 \%$ in the literature.(Huchon and Fauconnier, 2010)

Diagnosis was confirmed in 44 patients (44/51 - 86\%) in total who were operated with the preliminary diagnosis of torsion in all the age groups for the period covered by the study. We think that this high rate of confirmation of the preliminary diagnosis is that our hospital is a tertiary healthcare center and its experience in this area. Furthermore, while hemorrhagic infarct was seen in pathology results of none of the patients, hemorrhagic infarct was seen in 7 patients $(63 \%)$ in postmenopausal period. The reason for this is that surgical procedures are planned for later times in postmenopausal patients because of additional symptoms including nausea, vomiting and fever accompanying the imaging findings are absent in the postmenopausal period and there is no expectation of fertility. In parallel with this, the period between admission and operation in our study was the longest for the postmenopausal period.

The right ovary undergoes torsion more frequently than the left ovary. The reason for this is the greater length of the right utero-ovarian ligament and localization of the sigmoid colon at the left side (Huchon and Fauconnier, 2010). Consistently with the literature, torsion was observed in the right adnexa in 40 patients $(58.8 \%)$.

An adnexal mass lesion is present in the great majority of torsion cases in adults. It has been reported that no adnexal pathology is found only in 8 to $18 \%$ of cases. Majority of these masses are benign ovarian masses and tubal or paraovarian cysts (Anders and Powell, 2005; White and Stella, 2005). Cystic teratomas (60\%) and cystadenomas $(30 \%)$ constitute majority of benign ovarian masses (Oltmann et al., 2010). Torsion in ovaries in physiologic sizes is seen rarely. It is considered that adnexal torsion can be secondary to congenitally long ovarian ligament or extraordinarily loose pelvic ligaments in cases where there are no cysts or tumors (Bayer and Wiskind, 1994). In our study, no pathologies were found in 12 patients (48\%) in the adolescent period (48\%) and in 9 patients $(28.1 \%)$ in the reproductive period. The most frequent pathology in the adolescent $(5 / 25,20 \%)$ and reproductive $(7 / 32,21.9 \%)$ periods was mature cystic teratoma.

Malignancy potential and ovarian masses is an important issue in the management of patients diagnosed with torsion. No malignancy was found in several case series and studies in the pathology results after operations carried out because of torsion (Rody et al., 2002; Oelsner et al., 2003). Malignancy is extremely rare particularly in torsion cases in adolescent cases (0-3\%) (Savic et al., 2008). A possible explanation is that malignant lesions and endometriomas cause more fibrosis and leads to adhesions to the surrounding tissues, and torsion is seen less in such cases (Hibbard, 1985). The increased malignancy potential is obvious only in postmenopausal women, and malignancy rates reaching $22 \%$ has been reported in case series evaluating the postmenopausal patients diagnosed with adnexal torsion (Eitan, et al. 2007). In our study, $1(9.1 \%)$ malignant tumor is reported for the postmenopausal period, and no endometrioma was reported.

In our times, aggressive treatment approaches are less preferred in the treatment of adnexal torsion, and organ-preserving conservative procedures like detorsion are preferred in the first 
place. Long-term results of conservative treatment and more radical treatment methods are conflicted in the literature (Bellati et al., 2014; Parelkar, et al. 2014). Classical theory mentions increase in the thromboembolic event risk after detorsion, and as a possible reflection of this, preference by many gynecologists and obstetricians of oophorectomy in the treatment of torsion is between $30 \%$ and $86 \%$ (Rossi et al., 2012). Pulmonary embolism incidence in torsion cases is reported in studies as $0.2 \%$, while no differences have been reported reaching this rate after detorsion (McGovern et al., 1999). In our study also, no thromboembolic events were observed after conservative procedures.

\section{Conclusion}

Adnexal torsion is a clinical condition that will always be actual for gynecologists and obstetricians because of its prevalence and diagnostic difficulties. Currently, rapid management and laparoscopic and organpreserving approaches are important particularly for patients with fertility expectations. Although significant differences are not observed in clinical presentation and laboratory findings between age groups, mass lesions accompanying torsion and differences in pathologies of such masses must be kept in mind. While the clinical findings are nonspecific in the adolescent age group, suspected patients must be evaluated in detail for the sake of early diagnosis, and surgical intervention must be considered in the first place if the diagnosis of torsion cannot be eliminated and an early diagnosis is not possible with the purpose of preserving the ovarian reserve. Presence of a mass lesion accompanying the adnexal torsion must be searched for by all means in postmenopausal women, and pathology results of this mass must always be followed up because of the malignancy potential.

\section{Reference}

Albayram F, Hamper UM. Ovarian and adnexal torsion: spectrum of sonographic findings with pathologic correlation. Journal of Ultrasound in Medicine. 2001;20(10):1083-9.

Anders JF, Powell EC. Urgency of evaluation and outcome of acute ovarian torsion in pediatric patients. Archives of pediatrics \& adolescent medicine. 2005;159(6):532-5.

Bayer AI, Wiskind AK. Adnexal torsion: can the adnexa be saved? American journal of obstetrics and gynecology. 1994;171(6):1506-11.
Ethics Committee Approval: Ethics committee approval was received for this study from Clinical Research Ethics Committee of Liv Hospital Ankara

Peer-review: Externally peer-reviewed.

Author Contributions: Concept - B.Ç; DesignB.Ç; Supervision C.Ş; Materials -Bu. C, B.T; Data Collection and/or Processing - B.C, Bu. C; Analysis and/or Interpretation - F.K, Bu. C; Literature Review - B. C, Bu. T; Writing - B. C, F. K; Critical Review - Bu.C, C. S.

Conflict of Interest: No conflict of interest was declared by the author.

Financial Disclosure: The authors declared that this study hasn't received no financial support.

Bellati F, Ruscito I, Gasparri ML, Antonilli M, Pernice M, Vallone C, et al. Effects of unilateral ovariectomy on female fertility outcome. Archives of gynecology and obstetrics. 2014;290(2):349-53.

Bodur S, Alanbay I, Karasahin KE, Kinci MF, Fidan U, Keskin U. Chronic Ovarian Torsion Mimicking Subserosal Postmenopausal Fibroid. European Journal of Obstetrics and Gynecology and Reproductive Biology. 2016;206:e137.

Bronstein ME, Pandya S, Snyder CW, Shi Q, Muensterer OJ. A meta-analysis of B-mode ultrasound, Doppler ultrasound, and computed tomography to diagnose pediatric ovarian torsion. European Journal of Pediatric Surgery. 2015;25(01):82-6.

Chang S-D, Yen C-F, Lo L-M, Lee C-L, Liang CC. Surgical intervention for maternal ovarian torsion in pregnancy. Taiwanese Journal of Obstetrics and Gynecology. 2011;50(4):458-62.

Chiou S-Y, Lev-Toaff AS, Masuda E, Feld RI, Bergin D. Adnexal torsion: new clinical and imaging observations by sonography, computed tomography, and magnetic resonance imaging. Journal of Ultrasound in Medicine. 2007;26(10):1289-301.

Eitan R, Galoyan N, Zuckerman B, Shaya M, Shen $\mathrm{O}$, Beller $\mathrm{U}$. The risk of malignancy in postmenopausal women presenting with adnexal torsion. Gynecologic oncology. 2007;106(1):211-4.

Ekmekci E, Aydogmus H, Ergun Y, Eren R. Analyzing adnexiel torsion cases recognized in our clinic retrospectively. Turkiye Klinikleri Journal of Gnynecology and Obstetrics. 2010;20(5):287. 
Gunay T, Yardimci Od, Hocaoğlu M, Bör Ed, Erdem G. Ovarian Torsion and Surgical Treatment: 5 years experience of a tertiary center. Journal of Kahramanmaras Sutcu Imam University Faculty of Medicine.2018;13(2):3342.

Hibbard LT. Adnexal torsion. American journal of obstetrics and gynecology. 1985;152(4):456-61.

Houry D, Abbott JT. Ovarian torsion: a fifteenyear review. Annals of emergency medicine. 2001;38(2):156-9.

Huchon C, Fauconnier A. Adnexal torsion: a literature review. European Journal of Obstetrics \& Gynecology and Reproductive Biology. 2010;150(1):8-12.

Lomano jm, Trelford jd, Ullery jc. Torsion of the uterine adnexa causing an acute abdomen. Obstetrics \& Gynecology. 1970;35(2):221-5.

Mashiach R, Melamed N, Gilad N, Ben-Shitrit G, Meizner I. Sonographic diagnosis of ovarian torsion: accuracy and predictive factors. Journal of Ultrasound in Medicine. 2011;30(9):120510.

Mazouni C, Bretelle F, Menard J, Blanc B, Gamerre M. Diagnosis of adnexal torsion and predictive factors of adnexal necrosis. Gynecologie, obstetrique \& fertilite. 2005;33(3):102-6.

McGovern PG, Noah R, Koenigsberg R, Little AB. Adnexal torsion and pulmonary embolism: case report and review of the literature. Obstetrical \& gynecological survey. 1999;54(9):601-8.

Moribata Y, Kido A, Yamaoka T, Mikami Y, Himoto $\mathrm{Y}$, Kataoka M, et al. MR imaging findings of ovarian torsion correlate with pathological hemorrhagic infarction. Journal of Obstetrics and Gynaecology Research. 2015;41(9):1433-9.

Noviello C, Romano M, Papparella A, Ciavattini A, Martino A, Cobellis G. The isolated tubal torsion: an insidious pediatric and adolescent pelvic urgency. La Pediatria Medica e Chirurgica. 2018;40(2):48-51

Naiditch JA, Barsness KA. The positive and negative predictive value of transabdominal color Doppler ultrasound for diagnosing ovarian torsion in pediatric patients. Journal of pediatric surgery. 2013;48(6):1283-7.

Oelsner G, Cohen SB, Soriano D, Admon D, Mashiach S, Carp H. Minimal surgery for the twisted ischaemic adnexa can preserve ovarian function. Human Reproduction. 2003;18(12):2599-602.
Oltmann SC, Fischer A, Barber R, Huang R, Hicks B, Garcia N. Pediatric ovarian malignancy presenting as ovarian torsion: incidence and relevance. Journal of pediatric surgery. 2010;45(1):135-9.

Parelkar SV, Mundada D, Sanghvi BV, Joshi PB, Oak SN, Kapadnis SP, et al. Should the ovary always be conserved in torsion? A tertiary care institute experience. Journal of pediatric surgery. 2014;49(3):465-8.

Rody A, Jackisch C, Klockenbusch W, Heinig J, Coenen-Worch V, Schneider H. The conservative management of adnexal torsiona case-report and review of the literature. European Journal of Obstetrics \& Gynecology and Reproductive Biology. 2002;101(1):83-6.

Rossi BV, Ference EH, Zurakowski D, Scholz S, Feins NR, Chow JS, et al. The clinical presentation and surgical management of adnexal torsion in the pediatric and adolescent population. Journal of pediatric and adolescent gynecology. 2012;25(2):109-13.

Santra D, Dasgupta A, Ray N, Talukder A, Dasgupta S. Adnexal Torsion: Clinical Presentations and Challenges. Journal of Clinical \& Diagnostic Research. 2018;12(6).

Savic D, Stankovic ZB, Djukic M, Mikovic Z, Djuricic $S$. Torsion of malignant ovarian tumors in childhood and adolescence. Journal of Pediatric Endocrinology and Metabolism. 2008;21(11):1073-8.

Shadinger LL, Andreotti RF, Kurian RL. Preoperative sonographic and clinical characteristics as predictors of ovarian torsion. Journal of Ultrasound in Medicine. 2008;27(1):7-13.

White M, Stella J. Ovarian torsion: 10-year perspective. Emergency Medicine Australasia. 2005;17(3):231-7.

Wilkinson C, Sanderson A. Adnexal torsion-a multimodality imaging review. Clinical radiology. 2012;67(5):476-83. 